\title{
Integrated access control system for ports
}

\author{
F. Garzia ${ }^{1}$, E. Sammarco ${ }^{2} \&$ R. Cusani ${ }^{1}$ \\ ${ }^{1}$ INFOCOM Department, SAPIENZA - University of Rome, Italy \\ ${ }^{2}$ General Direction of Safety, Security and Civil Protection, \\ Vatican City State
}

\begin{abstract}
The security of a port is strongly dependent on the use of integrated access control technology systems. Any weakness of the integrated access control system involves a weakness of the port. For this reason it is necessary to design and realize highly integrated, efficient and reliable access control systems. The authors illustrate the work made to design and realize the integrated access control system of the most important commercial and tourist ports of Italy.
\end{abstract}

Keywords: access control system, integrated security system, security system.

\section{Introduction}

The activation of the new International Ship and Port Facility Security (ISPS) code forces all the ports to comply with the new prescriptions in matter of security and access control in ports. For this reason it has been necessary to design and develop an access control system that allows the port Authorities to acquire a technological system which respects the law prescriptions and optimizes, reduces and controls the boarding flows, offering, at the same time, a plenty of services such as the centralized check-in service for ship companies.

For this reason it has been necessary to design an automatic port areas access control system [1-9] capable of managing, as a component of an integrated security system, the passengers and goods flows without reducing the security standards but capable of reducing the access time relative to the older used system. In fact the designed system is capable of reaching opening time of the entrance bar and ticket printing that are lesser than 5 seconds.

The system is also endowed with automatic check-in functionalities, allowing the passengers to reach directly the boarding areas without passing through the ticket office, reducing and optimizing the vehicle traffic flows inside the port. 
The designed system is also opened towards other systems such as Ship Companies, Police, Sea Guard, Port Operators, etc., to allow a high level of integration of all the subjects involved in the control and boarding processes.

The scope of the paper is to illustrate the mentioned advanced integrated access control system, the difficulties found for its design and realization, and the results obtained, from its installation, in the normal and emergency situations.

Due to secrecy reasons, the integrated access control system is illustrated according to the general philosophy design, without illustrating specific details that could compromise the security of the system itself.

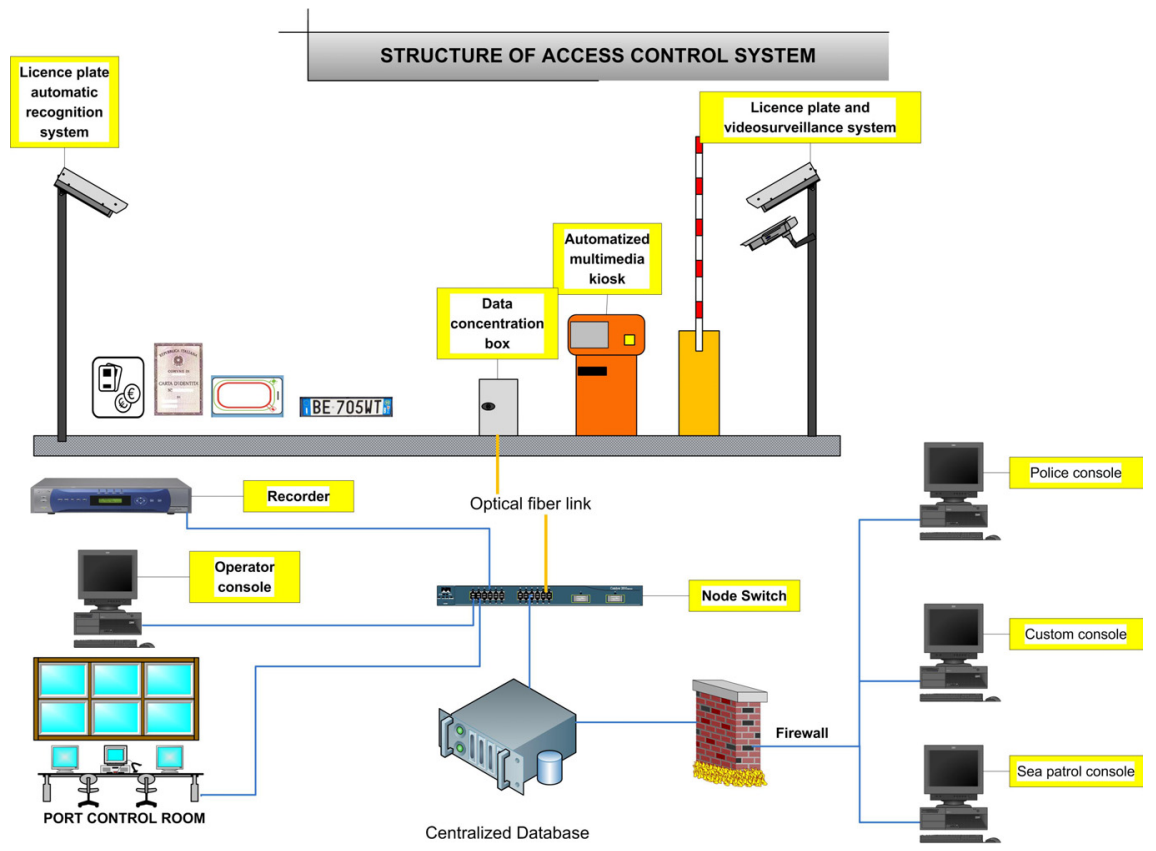

Figure 1: General scheme of the access control system.

\section{The access control system}

The system has been designed to comply with security and traffic procedures of the ports. For this reason it has been necessary to consider and respect a plenty of prescriptions and limitations imposed by the following elements:

1) international Ship and Port Facility Security code of December 2002;

2) interministerial committee for the maritime safety and security prescriptions of April 2004;

3) security evaluation document;

4) security plan of the port;

5) boarding areas and docks assignments; 
6) mean boarding time of each ship company;

7) position, inside the port, of ticket offices and entrance gates;

8) position of Lan/Wan network (optical fiber back bone) and design of the new one;

9) position of the wireless network and design of the new one;

10) network infrastructure and position of connection nodes;

11) traffic flows control video surveillance system;

12) licence plate recognition system of entrance gates;

13) access control multimedia kiosk endowed with automatic communication interface, Radio Frequency Identification Device (RFID) badge reader, ticket or temporary access printer, personal identification document reader, control camera.

The system allows to reach the following goals:

1) entrance/exit punctual control of all the vehicles in the port area;

2) entrance/exit punctual control of all the persons in the port area;

3) access control as a function of user profile inside the port;

4) information management database available for the security services;

5) dynamic parking areas management;

6) boarding area entrances management;

7) management of the information acquired;

8) real time data statistic of entrances, exits, parking according to different user profiles.

The entrance/exit punctual control of vehicles allows:

1) to have in real time all the information about the vehicles organized according to the typology or to the user profile (port worker, visitor, ship passenger, etc.);

2) the entrance of only authorized vehicles (licence plate recognition system);

3) to calculate the permanence time inside the port according to different user profiles;

4) to trace the vehicles transit inside the port.

The entrance/exit punctual control of person allows:

1) to have in real time all the information about the person according to the user profile (port worker, visitor, ship passenger, etc.);

2) the entrance of only authorized persons;

3) to calculate the permanence time inside the port according to different user profiles;

4) to trace the person transit inside the port.

The access control as a function of user profile inside the port allows of optimizing the entrance procedures creating proper preferential lanes inside the port according to the user profile.

The licence plate management by means of the security system allows:

1) real time recognition of licence plates;

2) creation of a database of undesired licence plates (black list);

3) creation of database of authorized licence plates (white list);

4) creation of a statistical and informative database of traffic flows. 
The dynamic parking area management allows:

1) to optimize the parking spaces inside the port;

2) to verify in real time the entrance of users to the dedicated areas.

The real time data statistic of entrance, exit and parking according to different user profiles allows one to manage the following data:

1) entrance according to user profile;

2) exit according to user profile;

3) parking according to user profile;

4) user profile present inside the port; and a lot of other data.

In the following the different subsystems of the access control system are illustrated.

\section{Telecommunication infrastructure}

The design of the system involved the design of a dedicated telecommunication system.

The telecommunication system is composed of nodes. Every node is independent and allows the connection of all the devices necessary to the access control system.

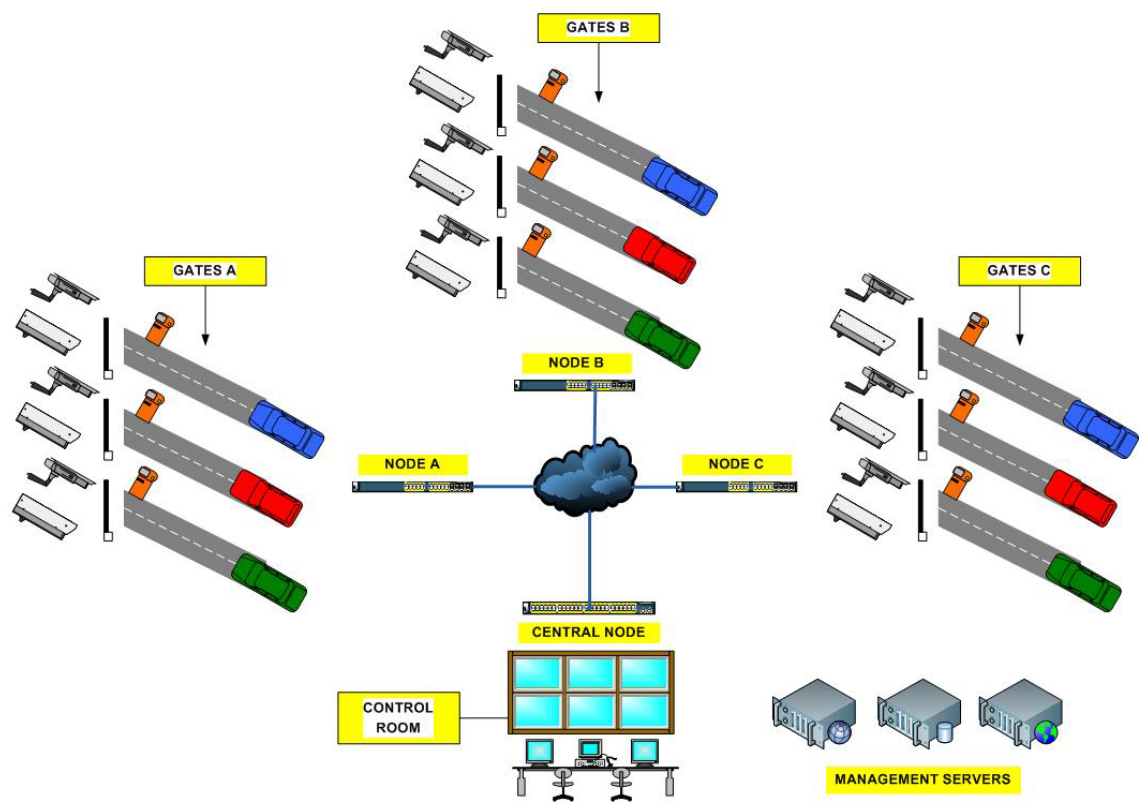

Figure 2: General scheme of the telecommunication system.

A proper virtual LAN is dedicated to each service inside the network, allowing to manage, in an equilibrate way, the band necessary to the communication. Nodes are connected each other by means of optical fibers. The 
connections are properly redundant to ensure a high reliability in the presence of an optical fiber damage. In some places it is not possible the optical fibers connection: in this case a broadband wireless connection was used, with a velocity equal to $300 \mathrm{Mb} / \mathrm{s}$.

All the hardware, software and network devices are placed in a proper dedicated cooled room, supplied by a proper power supplier capable of ensuring an autonomy of 1 hour in the absence of the main electrical supply.

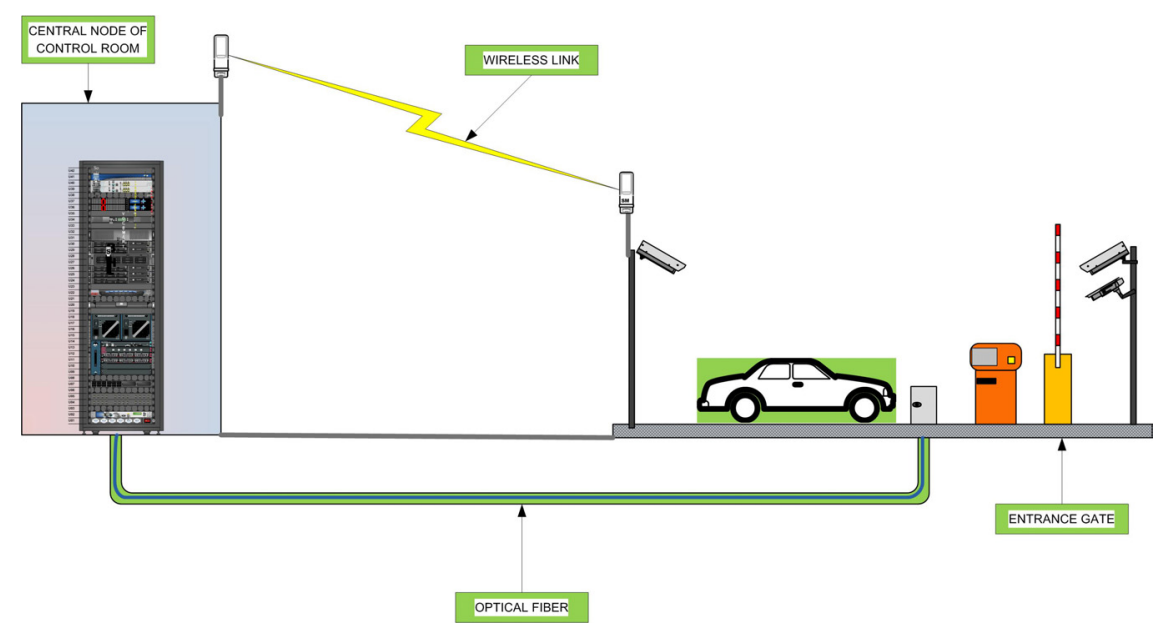

Figure 3: Connection modality of telecommunication system.

The design of server environment has been made to make them operate in fault tolerance modality to manage high traffic peaks even in the presence of malfunctioning of one or more than one of their component.

A proper routing policy has been developed to guarantee a high security level and a high flexibility to the whole system.

For this reason different devices capable of ensuring reliability and secure communication with the different check-in and ship company clients have been integrated in the system.

The passive routing devices are properly aided by load balance devices that allow one to share, in a smart way, the working duties between the different application servers.

All the software has been developed using international opened standards, basing on more diffused framework (J2EE, MS, NET). Data storing has been made using a DBMS relational database based on SQL standards. All the main functions of management and control are implemented on web interfaces. The web interfaces comply with normative concerning usability and accessibility. The communication and integration with other systems is based on SOAP (XML-RPC) protocol.

The software architecture is based on Model View Controller (MVC) paradigm realized by means of the design of a three-tier system that comply with 


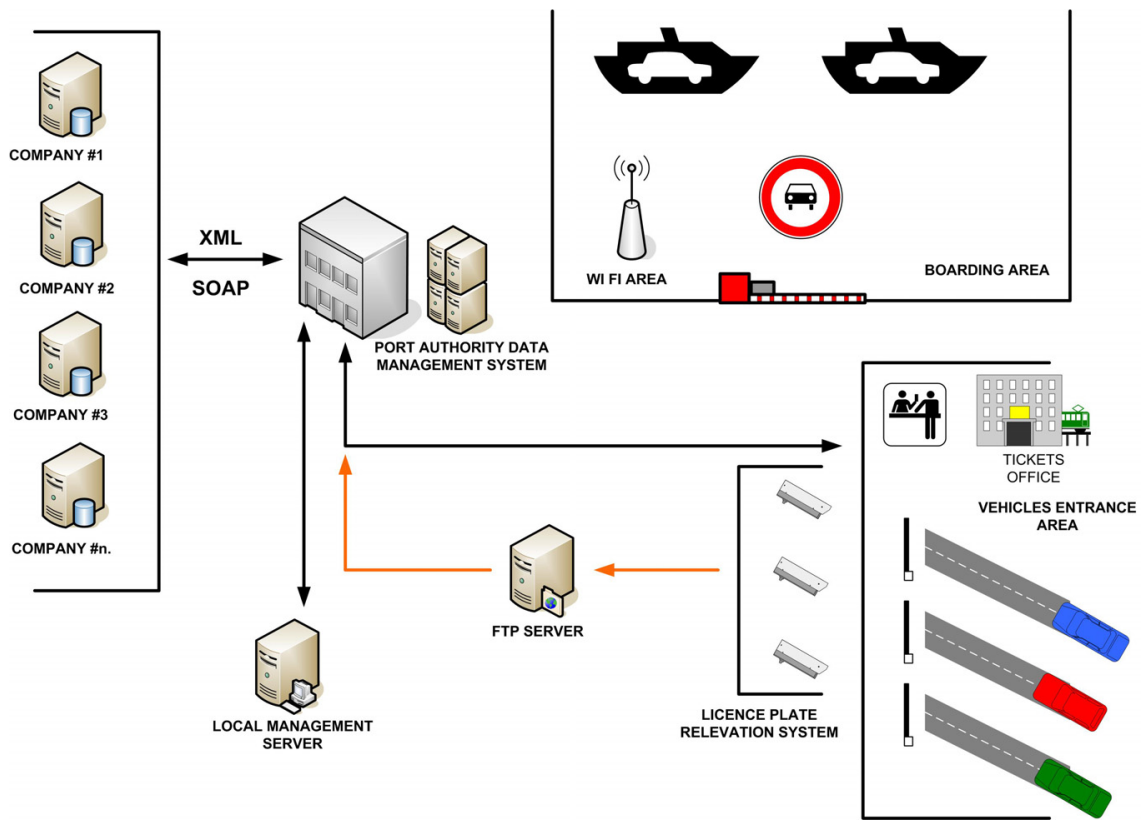

Figure 4: Scheme of the informative system.

W3C standard and oriented towards an approach based on Service Oriented Architecture (SOA) services.

The activity of installation and configuration of the whole applicative platform included the following phases:

1) realization of the central DBMS environment;

2) realization and installation of the security system integration software;

3) realization and installation of the integration software with access control system, licence plate recognition system, face recognition system, RFID badge reader;

4) realization and installation of centralized check-in software;

5) design and realization of the links with the enabled port operators;

6) design and realization of the links with ship companies.

In particular, the check-in software manages and redirects the XML requests and answers of the whole system and translate them in instructions for the boarding procedures (bar opening, card printing, access control, etc.).

The centralized check-in software implements the following functionalities:

1) companies' management;

2) users' management;

3) multimedia kiosks' management;

4) accesses control management;

5) remote configuration of entrance gates;

6) user permission management;

7) multimedia kiosks remote assistance management;

8) ship companies XML-SOAP links management; 
9) port operator external database ODBC link management;

10) ship companies XML link management;

11) logging access and system operation management;

12) docks and departures chart management.

Proper procedures for the integration with visual informative system of the port (public screens, etc.) have been implemented.

The system is also endowed with a proper web based client module that ticket offices of ship companies can use to print directly boarding card at the entrance gates.

\section{The gate system}

The entrance gate is designed to use a series of sensors necessary to acquire the information dedicated to the automatic management of itself. In the following the scheme of the entrance gate, together with sensors positioning, is shown.

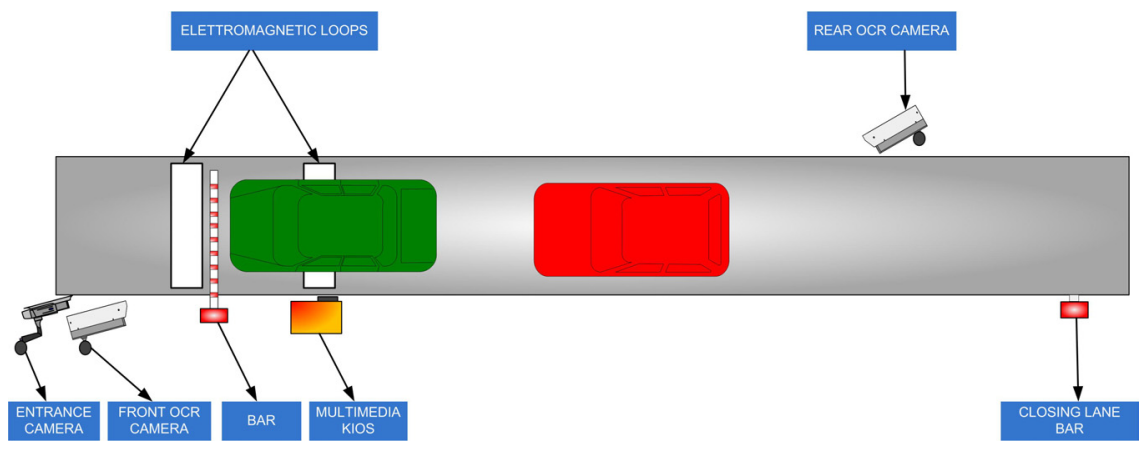

Figure 5: Scheme of the entrance gate.

Every lane uses a proper camera dedicated to the area analysis and to the image recording 24 hours a day and two further cameras dedicated to the licence plates recognition (Optical Character Recognition camera or OCR camera) that send acquired data directly to a proper multimedia kiosk.

The multimedia kiosk acquires data coming from OCR cameras and verifies the presence of the licence plate in a proper white list (authorized access), black list (unauthorized access) or passenger list. According to input data, it can generate an alarm, open the entrance bar or print the check-in ticket. It is also endowed with a RFID reader that can be used by port personnel.

The gates is also equipped with two electromagnetic loops, located close to the multimedia kiosk, that avoid the bar closing when a vehicle is in the middle.

\subsection{The licence plate recognition subsystem}

For licence plate recognition functionality new generation cameras have been used. They can acquire data related to high velocity traffic flows. The subsystem is placed in a proper enforced box located close to the entrance gate. 


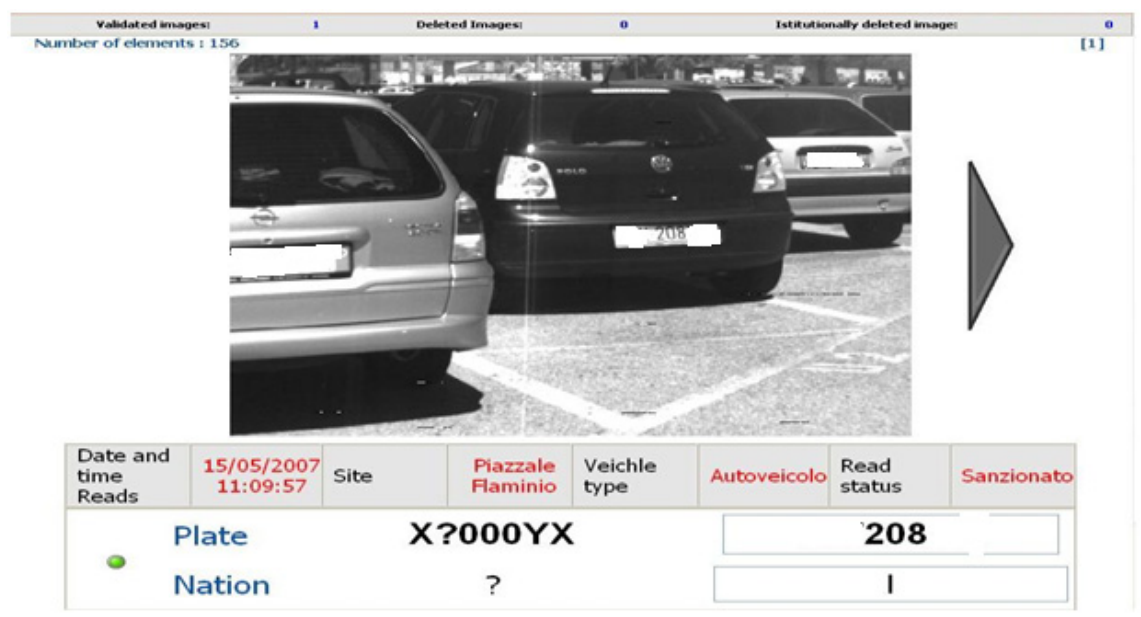

Figure 6: Example of licence plate recognition screen page of the system.

The OCR cameras embed all the hardware and software necessary to the licence plate recognition, ensuring a high recognition rate. They are capable of reading and recognizing the licence plates present in the scene without any triggering. They are also characterized by reduced dimension that greatly simplify their installation.

The OCR camera is constituted by a binocular optics where a black/white high-resolution element (1400 x 1024 pixels) and a colour high-resolution element (1400 x 1024 pixels) are installed. The processing unit is composed by a triple element (Floating Point Gate Array or FPGA, Digital Signal Processing or DSP and Central Processing Unit of CPU): the owner software, named $\mathrm{O}^{2} \mathrm{CR}$, works on the dedicated DSP unit. The OCR cameras are also equipped with infrared light to ensure a clear night vision. They communicate by means of a TCP/IP Ethernet 10/100 Mbps connection.

Thanks to the their computation capability, they are able of processing directly on board the licence plates data acquired, greatly improving the performances of the whole system.

The OCR cameras divide the recognition process in 3 phases managed properly by the three built-in components:

1) the FPGA executes a pre-processing of the images;

2) the DSP executes the real recognition of licence plate;

3) the CPU executes the management and communication of licence plates data.

The $\mathrm{O}^{2} \mathrm{CR}$ software can be configured to recognize, in an easy way by means of reading rules, the most of European and extra European licence plates.

In case of interruption of communication network, the system stores the data related to the licence plates and traffic flow of the gate and transmits them to the central database of the control room when the communication is restored. 


\subsection{The video surveillance subsystem}

The entrance gate are also equipped with a videosurveillance system that allows to monitor and record image using the the telecommunication network.

The system is based on TCP/IP network protocol to allow a more flexible management of itself. In fact, if the network is properly designed, it is possible in any moment to add or remove cameras without introducing further connection cable, ensuring a high modularity of the system.

The cameras send their images to the central control room using the fixed network or the wireless network. The images are stored, in a digital way, on proper digital recorders.

The system is composed by:

1) IP videocameras with power over ethernet and dome functionalities;

2) digital videorecording system;

3) management system.

\section{Further functionalities of the access control system}

The system ensures further functionalities that are illustrated in the following.

First of all it allows the communication between different ports so that the entrance data of the leaving port are sent to the arrival port to be used as exit data for people and vehicles.

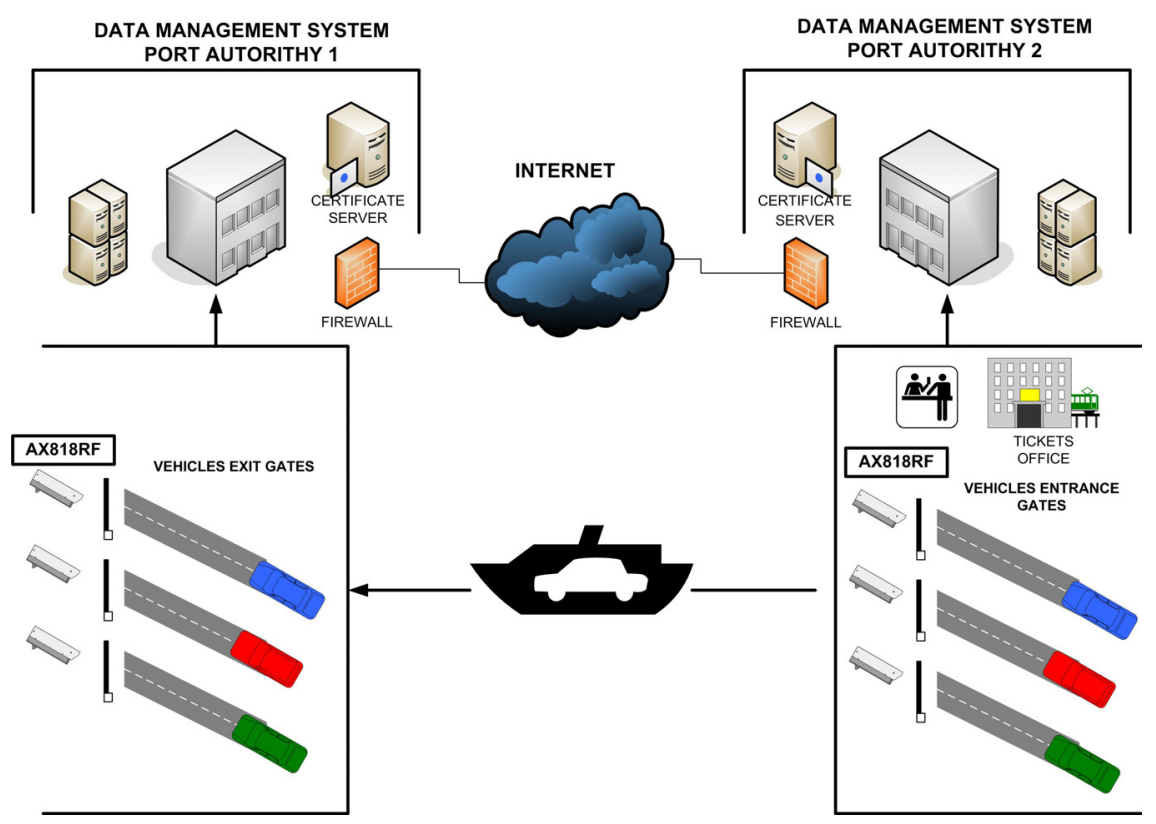

Figure 7: Scheme of the interconnection architecture between different ports. 
The system prints directly tickets for passengers and vehicles with the graphic layout of the required ship company, adding a proper bar code with all the necessary information.

The tickets contain also the data related to the vehicle (height and length) acquired by means of proper sensors installed at the entrance gates: these data are very useful to the ship companies to optimize the boarding operations.

The tickets are also endowed with a proper magnetic strip that allows them to be used as key of the cabin when they are on board on the ships.

The OCR cameras are capable of recognizing the nationality of vehicles and producing directly vocal messages in the desired language by means of the multimedia kiosks of the entrance gates.

The use of wireless networks allows the use of mobile devices that ensures a series of advanced services, very useful for port management. In fact, all the personnel is equipped with proper wireless terminals that allow them to manage and control all the access data from any place of the port. The ship company operators can read directly bar code tickets with their portable terminals and acquire immediately all the boarding information related to passenger and vehicles, reducing the boarding operations and the related time. The security personnel is equipped with proper wireless OCR cameras that read the licence plates during their patrolling operations inside the port and provide them all the data related to the user profile (authorized or unauthorized vehicle, time and data of entrance, parking time, etc.).

\section{Conclusions}

The security management in complex contests, such as the ports, needs a detailed risk analysis of menaces and dangers that must be faced and a correct study, design and realization of an efficient access control system that is capable of integrating the different security functionalities, ensuring the maximum reciprocal interaction of the different systems involved.

In this way it has been possible to realize a powerful and versatile integrated access control system that guarantees a high level of security services of the most important Italian ports.

\section{References}

[1] Waltz, E., "Information Warfare - Principles and operations", Artech House Publisher, Boston (USA), 1998.

[2] Denning, D. E., "Information Warfare and Security", Addison-Wesley, Boston (USA), 1999.

[3] Nichols, R.K. \& Lekkas, P.C., "Wireless Security: Models, Threats, and Solutions“, McGraw-Hill”, New York (USA),2002.

[4] Garzia, F., "The integrated safety/security system of the Accademia Nazionale dei Lincei at Corsini Palace in Rome", Proc. of International Conference on Integrating Historic Preservation with Security, Fire 
Protection, Life Safety and Building Management Systems, Rome (Italy), pp.77-99, 2003.

[5] Garzia, F. \& Veca, G. M., "Integrated security systems for hazard prevention, management and control in the Italian high speed train line", Risk Analysis III, WIT Press, Southampton (UK), pp.287-293, 2002.

[6] Antonucci, E., Garzia, F. \& Veca, G.M., "The automatic vehicles access control system of the historical centre of Rome", Sustainable City II, WIT Press, Southampton (UK), pp.853-861, 2002.

[7] Garzia, F., Sammarco, E. \& De Lucia, M., "The security telecommunication system of the Vatican City State", Risk Analysis IV, WIT Press, Southampton (UK), pp.773-782, 2004.

[8] Garzia, F., Sammarco, E., "The integrated security system of the Vatican City State", SAFE 2005, WIT Press, Southampton (UK), pp.391-403, 2005.

[9] Garzia, F., Sammarco, E., Cusani, R. "The integrated access control system of the Vatican City State", SAFE 2007, WIT Press, Southampton (UK), pp.431-440, 2007. 